\title{
Interaction of Bessel Light Beams with Epsilon-near-zero Metamaterials
}

\author{
Svetlana Kurilkina ${ }^{1}$, , Mohammed A Binhussain ${ }^{2}$, Vladimir Belyi ${ }^{1}$, Nikolai Kazak ${ }^{1}$ \\ ${ }^{1}$ B.I.Stepanov Institute of Physics, National Academy of Sciences of Belarus, Minsk, Belarus \\ ${ }^{2}$ The National Center for Building System KACST, Riyadh, Saudi Arabia \\ *corresponding author, E-mail: s.kurilkina@ifanbel.bas-net.by
}

\begin{abstract}
The article ex plores possibilities and conditions of generation of a new type of diffraction-free needle-like field Bessel plasmon polaritons (BPPs) with super narrow cone angle in an epsilon-near-zero metamaterial, surrounded by semi-infinite dielectric media. Correct analytical expressions are obtained and analyzed in detail for the electric and magnetic fields of BPPs formed inside and outside the metamateral slab.
\end{abstract}

\section{Introduction}

Recent advances in nanofabrication and developments in the theory of light-matter interaction have brought to life a new class of composite media, known as metamaterials (MMs). MMs offer new avenues for manipulation of light, lithography, lifetime engineering, high-resolution imaging [1-3]. One subclass of metamaterials is hyperbolic metamaterials (HMMs) i $\mathrm{n}$ which one of the diagonal permittivity tensor components is negative [4-6]. This results in a hyperbolic dispersion instead of elliptic one as in conventional dielectrics. The reason for HM Ms widespread interest is due to relative ease of nanofabrication, broadband non-resonant response, wavelength tunability and high figure of merit [7]. Hyperbolic metamaterials can be use d for a variety of applications from negative index waveguides to nanoscale resonators $[6,7]$.

In the approximation of the effective medium theory hyperbolic metamaterial can be considered as an unia xial uniform medium characterizing by the effect ive permittivities which are dependent on parameters of HMM. The conditions can be fulfilled when one of the effective permittivities is very small $(\approx 0)$. As established earlier [8, 9], such metamaterials, named as e psilon-near-zero metamaterials (ENZMs), display unique properties by interaction with a plane wave, for example, the possibility of canalization of ra diation. Recently such $\mathrm{m}$ edia were experimentally realized [10].

In 1987 Durnin suggested a new type of waves, the socalled Bessel light beams (BLBs); they are also referred to as diffraction-free beams [11-18]. The transverse profile of the amplitude of these beams is described by a B essel function of the first kind. In the domain of sp atial frequencies BLBs are represented as a superposition of plane waves with wave vectors which are wrapped around a conical surface having the cone angle $2 \gamma$. The main properties of Bessel light beams are the ability to keep the transverse size of the central lobe unchanged much longer than the Rayleigh range and to restore the wave front behind an obstacle. Owing to these features BLBs are promising for a number of applications, for example, for optical trapping and manipulation of microparticles and atoms, and $\mathrm{f}$ or technical diagnostics of subjects with a sub-wave resolution [19-22].

The authors of Ref. [ $\left.\begin{array}{ll}2 & 3-27\end{array}\right]$ theoretically and experimentally investigated evanescent BLBs formed in the condition of the internal total reflection in an optically less dense dielectric medium. These beams exponentially decay while moving off the surface but retain their origi nal transversal shape. In those investigations, of $\mathrm{p}$ articular interest was the structure of the central lobe of e vanescent BLBs. It is established that its diameter can be reduced to a nanosize value. This makes it possible to use e vanescent Bessel beams in optical microscopy [28].

But the evanescent BLBs investigated before possess an essential disadvantage, namely, they are weak, which causes the necessity of application of strong laser field $s$ for their generation. One of the ways of taking Bessel light field advantages for microscopy is the formation of Bessel plasmon polaritons (BPPs) [29, 30].

The present report considers the peculiarities of generation of Bessel plasmon polaritons in epsilon-near-zero metamaterials. Investigation of this problem attracts interest owing to a possibility to combine unusual features of BPPs and ENZMs.

The paper is structured as follows. In Section 2 the description is given of features of generation of Bessel plasmon polaritons in the symmetrical structure on the base of ENZM sl ab sandwiched between two sem i-infinite dielectrics. The behavior of electric and magnetic vectors in the structure is analyzed in detail. A conclusion is given in Section 3 .

\section{Bessel plasmons in epsilon-near-zero metamaterials}

We considered the hyperbolic metamaterial made of metallic nanocylinders periodically embedded in th $e$ 
dielectric template matrix, having the thickness $h$ (Fig.1), surrounded by an external isotropic medium with the dielectric permittivity $\varepsilon_{1}$ (for example, by air with $\varepsilon_{1}=1$ ). This composite can be made electrochemically.

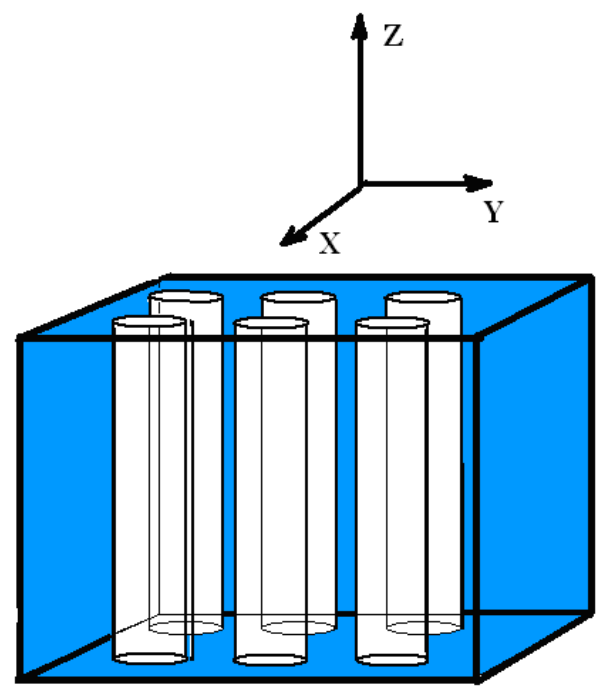

Figure 1: Metamaterial made of metallic nanocylinders periodically embedded in the dielectric template matrix.

At that, the controllable parameters are the metallic nanocylinder radius $r$, the metallic permittivity $\varepsilon_{m}$, the average distance between the ce nters of two a djacent cylinders $D$, and the membrane dielectric permittivity $\varepsilon_{d}$. It should be noted that permittivity of metallic nanocylinders is determined by the following correlation:

$$
\varepsilon_{m}(\lambda)=\varepsilon_{\infty}-\lambda^{2} /\left[\lambda_{p}^{2}\left(1+i \lambda \Gamma_{1} / 2 \pi c\right)\right],
$$

where $\varepsilon_{\infty}$ is the dielectric permittivity of the bulk metal, $\lambda$ is the wavelength of op tical radiation, $\lambda_{p}$ is the plasma wavelength, $\Gamma_{1} \approx \Gamma+V_{F} / 2 r, \Gamma$ is the damping constant, $V_{F}$ is the Fermi velocity. For silver (Ag) nanocylinders, for example,

have $\varepsilon_{\infty}=5 \quad, \quad \lambda_{p}=137 \mathrm{~nm} \quad, \quad \Gamma=32 \cdot 10^{12} \mathrm{~s}^{-1}$, $V_{F}=1.4 \cdot 10^{6} \mathrm{~ms}^{-1}[31]$.

In the approximation of the effective medium theory neglecting nonlocal spatial dispersion effects this metamaterial can be considered as a uniaxial uniform medium characterizing by $\mathrm{t}$ he following main effective permittivities [32]:

$$
\begin{aligned}
& \varepsilon_{x}=\varepsilon_{y}=\frac{\beta \varepsilon_{m} N+\varepsilon_{d}(1-N)}{\beta N+(1-N)}, \\
& \varepsilon_{z}=\varepsilon_{m} N+\varepsilon_{d}(1-N),
\end{aligned}
$$

where $N=\pi r^{2} / D^{2}$ is the inclusion factor $\beta=2 \varepsilon_{d} /\left(\varepsilon_{m}+\varepsilon_{d}\right)$. At that, $N<<1$ and $D, r<<\lambda$. As follows from Eq.(2), if the inclusion factor is fulfilled by the condition

$$
N=N_{0}=\varepsilon_{d} /\left(\varepsilon_{d}-\operatorname{Re} \varepsilon_{m}\right),
$$

the real part of the component $\varepsilon_{z}$ is equal to zero.

As it is known, the optical anisotropy of a uniaxial medium is characterized by the difference between two parameters: $\varepsilon_{o}=\varepsilon_{x}=\varepsilon_{y}$ and $\varepsilon_{e}$. At that:

$$
\begin{aligned}
& \varepsilon_{e}=\frac{\varepsilon_{o} \varepsilon_{z}}{\varepsilon_{o} \sin ^{2} \gamma_{e}+\varepsilon_{z} \cos ^{2} \gamma_{e}}, \\
& \gamma_{e}=\arcsin \frac{\sqrt{\varepsilon_{1}} \sin \gamma_{i n c}}{\sqrt{\varepsilon_{o}+\left(1-\frac{\varepsilon_{o}}{\varepsilon_{z}}\right) \varepsilon_{1} \sin ^{2} \gamma_{i n c}}},
\end{aligned}
$$

where $\gamma_{\text {inc }}$ is the angle of incidence of light. It follows from Eq.(3) that if $\varepsilon_{z}=0, \varepsilon_{e}$ is not equal zero only for $\gamma_{e}=0$. Moreover, from Eq. (5) one can see $\mathrm{t}$ hat for $\mathrm{t}$ he case $\varepsilon_{z}=0$ for the arbitrary $\gamma_{i n c}$ angle we have $\gamma_{e}=0$.

But it is very difficult to realize in practice the condition when $\varepsilon_{z}$ is exactly equal to zero. Fixing the value of permittivity $\varepsilon_{z}$ to a nonzero but low value, let us study the interaction of $\mathrm{TH}$ pol arized Bessel light beams having the wavelength $\lambda$ and the half-cone angle $\gamma_{i n c}$ with ENZM. From the Maxwell's equations for a uniaxial crystal in the cylinder coordinate system with the unit vectors $\vec{e}_{\rho}, \vec{e}_{\varphi}$, and $\vec{e}_{z}\left(\vec{e}_{z}\right.$ is collinear to the propagation direction) and $z=0$ at the entrance face of the metamaterial, one can obtain the formula for the longitudinal $(\mathrm{z})$, radial $(\rho)$ and azimuthal ( $\varphi$ ) components of the electric $\vec{E}$ and magnetic $\vec{H}$ fields for extraordinary $(e)$ type Bessel beams excited insi de semiinfinite ENZM :

$$
\begin{aligned}
& E_{\rho}^{e}=i \frac{k_{z e}}{\varepsilon_{o}} J_{m}^{\prime}(q \rho), \quad H_{\rho}^{e}=k_{0} \frac{m}{q \rho} J_{m}(q \rho), \\
& E_{\varphi}^{e}=-\frac{k_{z e}}{\varepsilon_{o}} k_{z} \frac{m}{q \rho} J_{m}(q \rho), \quad H_{\varphi}^{e}=i k_{0} J_{m}^{\prime}(q \rho), \\
& E_{z}^{e}=q \frac{1}{\varepsilon_{z}} J_{m}(q \rho), \quad H_{z}^{e}=0 .
\end{aligned}
$$

Here the phase $m$ ultiplier $\exp \left[i\left(k_{e} z+m \varphi\right)\right]$ is omitted; $k_{0}=2 \pi / \lambda ; q$ and $k_{z e}$ are transversal and longitudinal (in general case, complex) components of wave vectors forming BLB in the domain of spatial frequencies, respectively; $J_{m}(q \rho), J_{m}^{\prime}(q \rho)=\partial J_{m}(q \rho) / \partial(q \rho)$ are the $m$ order Bessel functions and their derivatives, $m$ is $\mathrm{t}$ he integer. At that

$$
\left.k_{z e}=\left[k_{0}^{2} \varepsilon_{o}-\left(\varepsilon_{o} / \varepsilon_{z}\right) q^{2}\right]^{1 / 2} . \quad(7) \quad\right)
$$

Note that the formula for TH BLB inside the sem iinfinite isotropic medium $\varepsilon_{1}$ can be obtained from Eqs.(6) where it shoul $\mathrm{d}$ be re placed $\varepsilon_{z} \rightarrow \varepsilon_{1}$, $k_{z e} \rightarrow k_{z 1}=\left[k_{0}^{2} \varepsilon_{1}-q^{2}\right]^{1 / 2}$.

The electric and magnetic vectors of the field inside the ENZM slab (it is denoted by the symbol " 2 ") of finite thickness $h$ and outside it in our case are expressed as 


$$
\begin{aligned}
& \vec{E}_{1,3}(R)=\vec{E}_{1,3}^{t r}(R)+\vec{E}_{1,3}^{l}(R), \\
& \vec{E}_{1}^{l}(R)=A_{\text {inc }} \frac{q}{\varepsilon_{1}} \exp i\left[m \varphi-k_{z 1} z\right] J_{m}(q \rho) \vec{e}_{z}, \\
& \vec{E}_{1}^{t r}(R)=-\frac{i A_{i n c} k_{z 1}}{\varepsilon_{1} \sqrt{2}} \exp i\left[(m-1) \varphi-k_{z 1} z\right] \vec{F}_{m}^{-}, \\
& \vec{E}_{3}^{l}(R)=A_{i n c} \frac{q}{\varepsilon_{1}} t \exp i\left[m \varphi+k_{z 3}(z-h)\right] J_{m}(q \rho) \vec{e}_{z}, \\
& \vec{E}_{3}^{t r}(R)=\frac{i A_{i n c} k_{z 1}}{\varepsilon_{1} \sqrt{2}} \exp i\left[(m-1) \varphi+k_{z 1}(z-h)\right] t \vec{F}_{m}^{-} . \\
& \vec{H}_{1}(R)=\frac{k_{0} A_{\text {inc }}}{\sqrt{2}} \exp i\left[(m-1) \varphi-k_{z 1} z\right] \vec{F}_{m}^{+}, \\
& \vec{H}_{3}(R)=\frac{k_{0} A_{i n c}}{\sqrt{2}} \exp i\left[(m-1) \varphi+k_{z 3}(z-h)\right] t \vec{F}_{m}^{+} \text {. } \\
& \vec{E}_{2}(R)=\vec{E}_{2}^{t r}(R)+\vec{E}_{2}^{l}(R), \\
& \vec{E}_{2}^{l, t r}(R)=\left(\vec{E}_{2}^{l, t r}\right)^{f}+\left(\vec{E}_{2}^{l, t r}\right)^{b}, \\
& \left(\vec{E}_{2}^{l}\right)^{f, b}=A_{\text {inc }} q \frac{1}{\varepsilon_{z}} s^{f, b} \exp i[m \varphi \pm \\
& \left. \pm k_{z e}|z-p h|\right] J_{m}(q \rho) \vec{e}_{z} \text {, } \\
& \left(\vec{E}_{2}^{t r}(R)\right)^{f, b}= \pm \frac{i A_{\text {inc }} k_{z e}}{\varepsilon_{o} \sqrt{2}} s^{f, b} \exp i[(m-1) \varphi \pm \\
& \left. \pm k_{z e}|z-p h|\right] \vec{F}_{m}^{-} \text {. } \\
& \vec{H}_{2}(R)=\left(\vec{H}_{2}(R)\right)^{f}+\left(\vec{H}_{2}(R)\right)^{b} \text {, } \\
& \left(\vec{H}_{2}(R)\right)^{f, b}=\frac{k_{0} A_{\text {inc }}}{\sqrt{2}} s^{f, b} \exp i[(m-1) \varphi \pm \\
& \left. \pm k_{z e}|z-p h|\right] \vec{F}_{m}^{+} .
\end{aligned}
$$

Here $R=(\rho, \varphi, z)$ are the cylindrical coordinates; $t$ is amplitude transmission coefficient of the slab, $s^{f}, s^{b}$ are the amplitude coefficients for the forward and backward Bessel fields inside the ENZM slab, respectively; $\quad \vec{F}^{ \pm}=J_{m-1}(q \rho) \vec{e}_{+} \pm J_{m+1}(q \rho) \exp (2 i \varphi) \vec{e}_{-} \quad ;$ $\vec{e}_{ \pm}=\left(\vec{e}_{1} \pm i \vec{e}_{2}\right) / \sqrt{2}$ are the unit circular vectors which are orthogonal to the $\vec{e}_{z}$ vector; $p=0$ for $s^{f}$ and $p=1$ for $s^{b}$; symbols "tr", "l" denote the transver sal and l ongitudinal component of the electric ( magnetic) vector, respectively; symbols " 1 " and " 3 " denote the media adjacent to the input and output surfaces of ENZM slab, respectively.

Using the boundary conditions of continuity of the tangential components of the electric and magnetic fields, which have to be satisfied at the planes $z=0$ and $z=h$, as well as Eq s. (8), (9), (10), (11), we obtain the system of equations for the coefficients $t, s^{f, b}$ from which i $\mathrm{t}$ follows:

$$
\begin{aligned}
& t=\left[\cos \left(k_{z e} h\right)-i \sin c\left(k_{z e} h\right) \frac{\left(\varepsilon_{o}^{2} k_{z 1}^{2}+\varepsilon_{1}^{2} k_{z e}^{2}\right) h}{2 \varepsilon_{o} \varepsilon_{1} k_{z 1}}\right]^{-1}, \\
& s^{f}=\frac{k_{z 1} \varepsilon_{o} t \exp \left(-i k_{z e} h\right)}{\varepsilon_{1} k_{z e}(1+\vartheta)},
\end{aligned}
$$

$s^{b}=-\frac{k_{z 1} \varepsilon_{o} t \vartheta}{\varepsilon_{1} k_{z e}(1+\vartheta)}$.

Here $\sin c\left(k_{z e} h\right)=\sin \left(k_{z e} h\right) /\left(k_{z e} h\right)$,

$\vartheta=\left[\left(k_{z 1} / \varepsilon_{1}\right)-\left(k_{z e} / \varepsilon_{o}\right)\right] /\left[\left(k_{z 1} / \varepsilon_{1}\right)+\left(k_{z e} / \varepsilon_{o}\right)\right]$.

From the boundary conditions for Eqs.(8), (9), (10), (11) one can find the dispersion equation determining the existence of Bessel surface $p$ lasmon polaritons in the structure shown in Fig. 1.

$\exp \left(i k_{z e} h\right)= \pm 1 / \vartheta$.

Note that Eq.(15) coincides with the condition of determining the poles of reflection coefficient:

$$
r=\frac{\vartheta\left(1-\exp \left(2 i k_{z e} h\right)\right)}{1-\vartheta^{2} \exp \left(2 i k_{z e} h\right)} .
$$

It is conveniently to rewrite Eq.(15) as a $\mathrm{n}$ equation for the unknown complex value $k_{z e}$ taking into account that $k_{z 1}^{2}=k_{0}^{2}\left(\varepsilon_{1}-\varepsilon_{z}\right)+\left(\varepsilon_{z} / \varepsilon_{o}\right) k_{z e}^{2}$. One ca $\mathrm{n}$ obtain from Eq. (15) that the condition of Bessel plasmon polariton generation in ENZM is fulfilled for $k_{z e} \approx 0$.

It follows from Eq.(7) that it is realized if the following condition is fulfilled:

$$
q=k_{0} \sqrt{\varepsilon_{z}} .
$$

At that,

$$
\begin{aligned}
& \operatorname{Re} q=\frac{k_{0}}{\sqrt{2}}\left[\operatorname{Re} \varepsilon_{z}+\sqrt{\left(\operatorname{Re} \varepsilon_{z}\right)^{2}+\left(\operatorname{Im} \varepsilon_{z}\right)^{2}}\right]^{1 / 2} \\
& \operatorname{Im} q=\frac{k_{0} \operatorname{Im} \varepsilon_{z}}{\sqrt{2}}\left[\operatorname{Re} \varepsilon_{z}+\sqrt{\left(\operatorname{Re} \varepsilon_{z}\right)^{2}+\left(\operatorname{Im} \varepsilon_{z}\right)^{2}}\right]^{-1 / 2}
\end{aligned}
$$

If $\operatorname{Re} \varepsilon_{z} \rightarrow 0$ we have $\operatorname{Re} q \approx \operatorname{Im} q=k_{0} \sqrt{\operatorname{Im} \varepsilon_{z}} / \sqrt{2}$. Then, if th e absorption of $m$ etal component of ENZM, determining the $\mathrm{v}$ alue of $\operatorname{Im} \varepsilon_{z}$, decreases, the parameter $\operatorname{Re} q$ decreases too. Note that $\operatorname{Re} q$ characterizes the halfcone angle of wave vectors $\gamma_{i n c}$ forming incident BLB in the domain of spatial frequencies.

If the condition $\operatorname{Im} \varepsilon_{z} \rightarrow 0$ is fulfilled, the generation of BPPs is observed at incidence on ENZM slab of Bessel light beam with the half-cone angle $\gamma_{i n c}=\arcsin \left(\operatorname{Re} q /\left(k_{0} \sqrt{\varepsilon_{1}}\right)\right) \rightarrow 0$.

We can represent dielectric permittivity $\varepsilon_{z}$ in the form

$$
\varepsilon_{z}=\left(\operatorname{Re} \varepsilon_{m}-\varepsilon_{d}\right) \alpha N_{0}+i \operatorname{Im} \varepsilon_{m}(1+\alpha) N_{0},
$$

where $\alpha=\Delta N / N_{0}, \Delta N$ is deviation of inclusion factor from $N_{0}$. As follows from Eqs. (17), (20), parameter $\operatorname{Re} q$ is dependent on the deviation of inclusion factor $\Delta N$. The $\gamma_{\text {inc }}(\Delta N)$ for the case of ENZM slab made on the base of alumina oxide with $\mathrm{p}$ eriodically embedded silver nanocylinders is represented in Fig. 2 It is seen that in real situation (when $\operatorname{Im} \varepsilon_{z} \neq 0$ ) the value of $\gamma_{i n c}$ is not equal zero too and decreases with increasing $\Delta N$. 


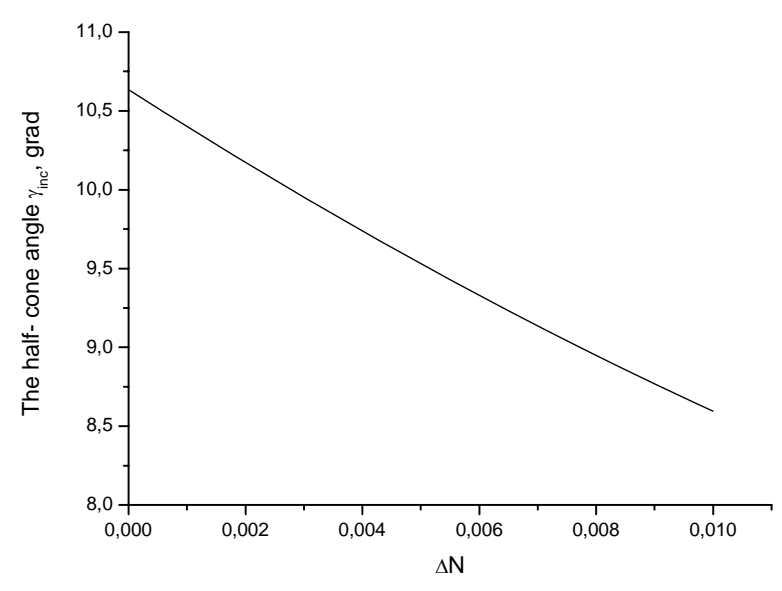

Figure 2: The dependence on the deviation of inclusion factor $\Delta N$ of the half-cone angle of incident Bessel light beam for which Bessel plasmon polariton is excited in ENZM slab made of alumina oxide with periodically embedded silver nanocylinders $(r=25 \mathrm{~nm})$ and surrounded by air. Here $\lambda=633 \mathrm{~nm}$.

It is in teresting to ph ysically interpret Bessel $\mathrm{p}$ lasmon polariton. As it $\mathrm{m}$ entioned above, Bessel b eam can be considered as a sup erposition of $p$-polarized plane monochromatic waves having the wavelength $\lambda$ and wave vectors lying on the surface of a cone. $\mathrm{E}$ very $p$-polarized plane-wave component of the incident BLB in conditions of the plasmon resonance excites the surface plasmon polariton (SPP) propagating along the interface of ex ternal medium and ENZM slab. Here the phase of every SPP is determined by the phase of the plane-wave component of the incident BLB. Thus, in the ENZM slab an array of propagating SPPs arises, wave vectors of which are being oriented in the direction of the center determining the intersection of the incident BLB axis with the surface of the epsilon-near-zero metamaterial. As a result there occurs the generation of pairs of two counter-propagating SPP waves with the wave vectors $\pm \vec{q}$. The generated SPPs will propagate in all the possible radial directions to form a localized SPP field. This results in a complex high-symmetric interference light structure emerging in sections parallel to the dielectricmetamaterial surface.

For the case where the longitudinal components of the electric field $\vec{E}$ for every radially propagating SPP has the same phase, in the center of such a standing light structure a maximum appears, i.e. the s hape of the $\vec{E}$ component of localized Bessel plasmon polariton is described by zeroorder Bessel function $J_{0}(q \rho)$. In a special case, each pair of counter propagating SPPs is in counter-phase and the local plasmonic field appears with a minimum in the ce nter, i.e. the so-called vortex localized BPP is formed. In this case the electric field $\vec{E}$ of vortex BPP is described by $J_{m}(q \rho)$ Bessel function.
One of most important characteristics of Bessel plasmon polariton is the first ring radius $R_{1} \approx 2.4 /(\operatorname{Re} q)$ in transversal section of intensity distribution. It follows from Eq.(18) that if $\operatorname{Im} \varepsilon_{z} \rightarrow 0$ then $R_{1} \rightarrow \infty$. But existence of absorption of metal component of ENZM limits the value of $R_{1}$.

The problem of attenuation of the BPP should be studied now. Let us consider this field outside the ENZM layer (at the interface between air and ENZM). With this aim the limited narrow Bessel light beam of radius $r_{0}$ in the transversal section should be considered. As follows from Eq. (8), the transversal distribution of the longitudinal component of the electric field of Bessel SPP is determined by the $J_{m}(q \rho)$ Bessel function which can be represented as a sum of the cylindrical Hankel functions of the first $H_{m}^{(1)}(q \rho)$ (outgoing) and second $H_{m}^{(2)}(q \rho)$ (incoming) kinds [33]:

$$
J_{m}(q \rho)=\left(H_{m}^{(1)}(q \rho)+H_{m}^{(2)}(q \rho)\right) / 2 .
$$

According to Eq.(21), in the region $\rho<r_{0}$ the BPPs are formed by converging and diverging conical beams described by appropriate Hankel functions. Outside the area $\rho>r_{0}$ there exists only a diverging conical beam, and that is why in the decomposition Eq. (21) it is sup posed that $H_{m}^{(2)}=0$. Using the asymptotic approximation of Hankel function [34], we obtain that the intensity of the longitudinal component of Bessel SPP electric vector $\left|\vec{E}^{l}\right|^{2} \sim\left|J_{m}(q \rho)\right|^{2}$ is determined by the expression:

$$
\left|\vec{E}^{l}\right|^{2} \sim \frac{1}{|q|^{2} \rho^{2}} \exp [-2(\operatorname{Imq}) \rho] .
$$

Thus, from Eq. (22) it follows that beyond the boundary of the exciting source the BPP decays exponentially in the radial direction. The $1 / \mathrm{e}$ energy-attenuation radius $R_{B S S P}$ is determined by the expression

$$
R_{B S S P}=1 /(2 \operatorname{Im} q) \text {. }
$$

Let us analyze in detail the features of Bes sel plasmon polariton generating in the structure represented in Fig.1. As it follows from Eq. (8) the electric (magnetic) vector of the field of BPP in dielectric medium near the exit surface of ENZM slab is d ependent on the transmission coefficient $t$ determining by the following expression at $k_{z e} \approx 0$ :

$$
t=\left[1-i \frac{\varepsilon_{o} k_{z 1} h}{2 \varepsilon_{1}}\right]^{-1} .
$$

It is evident from Eq. (24) that the value $t$ (and hence, the longitudinal and transversal components of electric vector of BPP inside the external medium " 3 ") is not equal to zero and dependent on the optical properties of ENZM and its thickness.

Much interest is attracted to investigate the features of BPP inside the ENZM slab. As it fo llows from Eqs. (10), (13), (14) in the conditions of plasmon resonance $k_{z e} \approx 0$ 
the longitudinal component of electric vector of BPP inside the ENZM slab is determined by the expression:

$$
\begin{aligned}
& \vec{E}_{2}^{l}(R) \approx i A_{\text {inc }} \frac{q}{\varepsilon_{\mathrm{z}}} \frac{k_{z 1} \varepsilon_{o} t}{\varepsilon_{1}}(z-h) \times \\
& \times \sin c\left[k_{z e}(z-h)\right] \exp i[m \varphi] J_{m}(q \rho) \vec{e}_{z} \approx \\
& \approx(-1+i) A_{i n c} \frac{k_{0}}{\sqrt{2 \operatorname{Im} \varepsilon_{z}}} \frac{k_{z 1} \varepsilon_{o} t}{\varepsilon_{1}}(z-h) J_{m}(q \rho) \vec{e}_{z} .
\end{aligned}
$$

Here $0<z<h$. It follows from Eq. (25) that the longitudinal component of electric vector of Bessel plasmon polariton decreases linearly with increasi ng the distance inside the slab. The intensity of longitudinal component is found from the expression

$$
I^{l} \sim \frac{k_{0}^{2}}{\operatorname{Im} \varepsilon_{z}} \frac{k_{z 1}^{2} \varepsilon_{o}^{2}|t|^{2}}{\varepsilon_{1}^{2}}(h-z)^{2}\left|J_{m}(q \rho)\right|^{2} .
$$

The value $I^{l}$ is equal to zero at $z=h$. Note that $I^{l}$ is greater for the case of ENZM with sm all absorption (small value of $\operatorname{Im} \varepsilon_{z}$ ) of the metal nanocylinders.

One can see from Eq. (26) that the transversal distribution of $I^{l}$ is described by the Bessel function. Then the size of the central lobe of intensity pattern is not changed moving off the entrance surface of the ENZM slab.

The transversal component of the electric vector of BPP inside the ENZM slab is given by

$$
\vec{E}_{2}^{t r}(R) \approx-A_{i n c} \frac{k_{z 1} t}{\sqrt{2} \varepsilon_{1}} \sin \left[k_{z e}(z-h)\right] \exp i[(m-1) \varphi] \vec{F}_{m}^{-},
$$

and it is very small.

As it follows from Eq. (11), the transversal component of magnetic vector can be expressed as

$$
\begin{aligned}
& \vec{H}_{2}(R) \approx \frac{i k_{0} A_{i n c} k_{z 1} \varepsilon_{o} t(z-h)}{\sqrt{2} \varepsilon_{1}} \times \\
& \times \sin c\left[k_{z e}(z-h)\right] \exp i[(m-1) \varphi] \vec{F}_{m}^{+} \approx \\
& \approx \frac{i k_{0} A_{i n c} k_{z 1} \varepsilon_{o} t(z-h)}{\sqrt{2} \varepsilon_{1}} \exp i[(m-1) \varphi] \vec{F}_{m}^{+} .
\end{aligned}
$$

One can see from Eq. (28) that $\vec{H}_{2}(R)$ has essential value.

It is important to emphasize that in opposite to the case of generation of Bessel plasmon polariton in metal film [35] in considered case only single BPP is formed inside the ENZM slab. The full field of Bessel plasmon polariton inside and outside the ENZM slab is described by the Eqs. (8), (9), (26), (27), (28).

\section{Conclusions}

Thus, in this paper a theory is developed of generation of Bessel plasmon polaritons in the structure including a epsilon-near-zero metamaterial layer sep arating semiinfinite dielectrics.

The comparison was made of Bessel plasmon polaritons investigated in this pa per and tra ditional surface plasmon polaritons. The traditional SPP is a propagating wave on the ENZM/dielectric surface. Unlike it, BPP is a superposition of counter propagating SPPs in all the possible radial directions. It is a complex high-symmetric interference light structure in sections parallel to the ENZM/d ielectric interface. It should be noted as opposed to the propagating surface plasmon polariton, the Bessel one is a standing light structure.

The problem is stud ied of attenuation of the Bessel plasmon polariton excited by a limited narrow Bessel beam. It is shown that outside the region of the exciting source the BPP decays exponentially in the radial direction.

We have analyzed the cases of symmetric "dielectric ENZM slab - dielectric" struct ure. A dispersion equation has been derived and analyzed for this case. The possibility is shown of excitation of the single type of Bessel plasmon polariton independently on the thickness of the ENZM slab. It is established that this BPP is c haracterized by essential transversal component of magnetic vector and longitudinal component of electric vector. The magnitude of the latter component is substantially dependent on the absorption in the metal nanocylinders.

The intensity distribution of the longitudinal component of the electric vector of Bessel plasmon polariton inside the ENZM layer is analyzed. It is shown that the large central lobe of the intensity pattern is not changed in its transversal size when moving off the entrance surface of the e psilonnear-zero metamaterial slab. So, it is established the possibility to form the diffraction-free needle-like standing plasmon field inside the ENZM layer.

The results obtained can be used for the development and optimization of techniques and devices for testing the quality of the surface of various substrates by Bessel plasmon polaritons.

\section{Acknowledgements}

The authors would like to thank King Abdul-Aziz City for Science and Technology (KACST) for Research Grant No. 814-33.

\section{References}

[1] J.B. Pendry, Negative refraction makes a p efraction makes a perfect lens, Phys. Rev. Lett. 85: 3966-3969, 2000.

[2] N. Fang, H. Lee, C. Sun, X. Zhang, Sub-diffractionlimited optical imaging with a silver superlens, Science. 308: 534-537, 2005.

[3] J.B. Pendry, D. Schurig, D.R. Smith, Controlling Electromagnetic Fields, Science 312: 1780-1782, 2006.

[4] D.R. Smith, P. Kolinko, D. Schurig, Negative refraction in indefinite media, J. Opt. Soc. Am. B21: 1032-1043, 2004.

[5] P.A. Belov, Y. Hao, Subwavelength imaging at optical frequences using a transmission device formed by a periodic layered metal-dielectric structure operating in the canalization regime, Phys. Rev. B73: 113110, 2006.

[6] V. P. Drachev, V. A. Podolsky, A.V.Kildishev, Hyperbolic metamaterials: new physics behind a 
classical problem, Opt. Express. 21: 15048-15064, 2013.

[7] A. J. H offman, L. Alekseyev, C.Gmachl, Negative refraction in sem iconductor metamaterials, Nature Materials 6: 946-950, 2007.

[8] G. Castaldi, S. Savoia, V. Galdi, Analytical study of subwavelength imaging by uniaxial epsilon-near-zero metamaterial slabs, Phys. Rev. B86: 115123, 2012.

[9] B. Wang, K.M. Huang, Shaping the radiation pattern with $\mathrm{Mu}$ and epsilon-near-zero metamaterials, PIER. 106:107-119, 2010.

[10] J. Gao et al, Experimental realization of epsilon-nearzero metamaterial slabs with $\mathrm{m}$ etal-dielectric multilayers, Appl. Phys. Lett. 103: 051111 (2013).

[11]J. Durnin, Exact so lutions for nondiffracting beams. I. The scalar theory, J.Opt.Soc.Am. A4: 651-654, 1987.

[12] J. Durnin, J.J . Muceli, J. H. Eberly, Diffraction-free beams, Phys. Rev. Lett. 58: 1499-1501, 1987.

[13]P. Sprangle, B. Hafizi, Comment on nondiffracting beams , Phys. Rev. Lett.: 66: 837-839, 1991.

[14]Z. Bouchal, J. Wagner, M. Chlup, Self-reconstruction of a distorted nondiffracting beam, Opt. Commun. 151: 207-211, 1998.

[15] Y. Lin et al, Experimental investigation of Bessel beam characteristics, Appl. Opt., 31: 2708-2713, 1992.

[16]D. McGloin, K. Dholakia, Bessel beams: diffraction in a new light, Contemp. Phys. 46: 15-28, 2005.

[17] J. Turunen, A.T. Friberg, Self-imaging and propagationinvariance in electromagnetic fields, Pure Appl. Opt. 2: 51-60, 1993.

[18]R. Horak, Z. Bouchal, J. Bajer, Nondiffracting stationary electromagnetic field, Opt. Commun. 133: 315-327, 1997.

[19]G. Milne, K. Dholakia, D. McGloin, K. VolkeSepulveda, P. Zemanek, Particle dynamics in a Bessel beam, Opt. Express. 15: 13 972- 13 987, 2007.

[20] V. Garcés-Chavéz, D. Roskey, M.D. Summers, H. Melville, D. McGloin, E.M. Wright, K. Dholakia, Optical levitation in a Bessel lig ht beam, Appl. Phys. Lett. 8: 4001-4003, 2004.

[21]L. Paterson, E. Pap agiakoumou, G. Milne, V.GarcésChavéz, T. Briscoe, W. Sibbett, L. Dholakia, A. Riches, Passive optical separation with a 'nondiffracting' light beam, J. Biomed. Opt. 12: 054017, 2007.

[22]T. Cižmár, V. Ga rcés - Chávez, K. Dholakia, P. Zemánek, Optical conveyor belt for delivery of submicron objects, Appl. Phys. Lett. 86: 101-1-174101-3, 2005.

[23] S. Rushin, A. Leizer, Evanescent Bessel beams, J. Opt. Soc. Am..A15: 1139-1143, 1998.

[24] S. N. Kurilkina, V.N. Belyi, N.S. Kazak, Features of evanescent Bessel light beams formed in structure $\mathrm{s}$ containing a dielectric layer, Opt. Comm. 283: 38603868, 2010.
[25] Q.Zhan, Evanescent Bessel beam generation via surface plasmon resonance excitation by a radially polarized beam, Opt.Lett. 31: 1726-1728, 2006.

[26] Muhanna K Al-Muhanna, S. N. Kurilkina, V. N. Belyi, N. S. Kazak, Energy flow patterns in an optical field formed by a su perposition of evanescent Bessel light beams, J.Opt. 13: 105703, 2011.

[27] H.Kano, D. Nomura, H. Shibuya, Excitation of surfaceplasmon polaritons by use of a zer oth-order Bessel beam, Appl. Opt. .43: 2409-2411, 2004.

[28] T. Grosjean, D. Courjon, D. Van Labeke, Bessel beams as virtual tips for near-field optics, J. Microscopy. 210: 319-323, 2003.

[29] H.Kano, D. Nomura, H. Shibuya, Excitation of surfaceplasmon polaritons by use of a zer oth-order Bessel beam, Appl. Opt. 43: 2409-2411, 2004.

[30] J. Zapata-Rodriguez et al, Nondiffracting Bessel plasmons, Opt. Express. 19: 19572-19581, 2011.

[31]W. Cai, V. Shalaev, Optical Metamaterials. Fundamentals and Applications, Springer, New York, 2010.

[32] R.Atkinson et al, Anisotropic optical properties of arrays of gold nanorods embedded in alumina, Phys. Rev. B73: 235402-1 - 235402-8, 2006.

[33]G.N. Watson, A Threatise on the theory of Bessel functions, Cambridge University Press, Cambridge, 1966.

[34] G. B. Arfken, H.J.Weber, F.E.Harris, Mathematical methods for physicists, Academic Press, Orla ndo FL., 1985.

[35] S. N. Kurilkina, V. N. Belyi, N. S. Kazak, Features of vortex Bessel plasmons generated in metal-dielectric layered structures, J. Opt. 15: 044017, 2013. 